This item was submitted to Loughborough's Research Repository by the author.

Items in Figshare are protected by copyright, with all rights reserved, unless otherwise indicated.

\title{
Vibration characteristics of MR cantilever sandwich beams: experimental study
}

PLEASE CITE THE PUBLISHED VERSION

http://dx.doi.org/10.1088/0964-1726/19/1/015005

PUBLISHER

(c) IOP Publishing Ltd

VERSION

SMUR (Submitted Manuscript Under Review)

LICENCE

CC BY-NC-ND 4.0

\section{REPOSITORY RECORD}

Prieto, Vianney Lara, Robert M. Parkin, Michael R. Jackson, Vadim V. Silberschmidt, and Zbigniew Kesy. 2019. "Vibration Characteristics of MR Cantilever Sandwich Beams: Experimental Study". figshare. https://hdl.handle.net/2134/14822. 
This item was submitted to Loughborough's Institutional Repository (https://dspace.lboro.ac.uk/) by the author and is made available under the following Creative Commons Licence conditions.

\section{creative
commons}

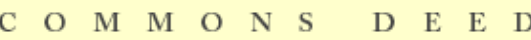

Attribution-NonCommercial-NoDerivs 2.5

You are free:

- to copy, distribute, display, and perform the work

Under the following conditions:

Attribution. You must attribute the work in the manner specified b the author or licensor.

Noncommercial. You may not use this work for commercial purposes.

No Derivative Works. You may not alter, transform, or build upon this work.

- For any reuse or distribution, you must make clear to others the license terms of this work.

- Any of these conditions can be waived if you get permission from the copyright holder.

Your fair use and other rights are in no way affected by the above.

This is a human-readable summary of the Leqal Code (the full license).

\section{Disclaimer 만}

For the full text of this licence, please go to: http://creativecommons.org/licenses/by-nc-nd/2.5/ 


\title{
Vibration characteristics of MR cantilever sandwich beams: Experimental study
}

\author{
Vianney Lara-Prieto $^{1}$, Rob Parkin ${ }^{1}$, Mike Jackson ${ }^{1}$, Vadim Silberschmidt ${ }^{2}$ and Zbigniew \\ Kęsy $^{3}$ \\ ${ }^{1}$ Mechatronics Research Group, Wolfson School of Mechanical and Manufacturing Engineering, \\ Loughborough University, UK \\ ${ }^{2}$ Mechanics of Advanced Materials, Wolfson School of Mechanical and Manufacturing Engineering, \\ Loughborough University, UK \\ ${ }^{3}$ Institute of Applied Mechanics, Technical University of Radom, Poland
}

\begin{abstract}
.
The concept of vibration controllability with smart fluids within flexible structures has been in the centre of interest in the past two decades. Although much research has been done on structures with embedded electrorheological (ER) fluids, there has been little investigation of magnetorheological (MR) fluid adaptive structures. In particular, a body of research on experimental work of cantilever MR beams is still lacking. This experimental study investigates controllability of vibration characteristics of magnetorheological cantilever sandwich beams. These adaptive structures are produced by embedding an MR fluid core between two elastic layers. The structural behaviour of the MR beams can be varied by applying an external magnetic field to activate the MR fluid. The stiffness and damping structural characteristics are controlled, demonstrating vibration suppression capabilities of MR fluids as structural elements. MR beams were fabricated with two different materials for comparison purposes. Diverse excitation methods were considered as well as a range of magnetic field intensities and configurations. Moreover, the cantilever MR beams were tested in horizontal and vertical configurations. The effects of partial and full activation of the MR beams were outlined based on the results obtained. Controllability of the beam's vibration response was observed in the form of variations in vibration amplitudes and shifts in magnitudes of the resonant natural frequency.
\end{abstract}

\section{Introduction}

One of the main issues in various structures is the undesirable excessive vibration. The control of structural vibrations can be implemented different ways such as modifying stiffness, mass, damping and shape, and by providing passive or active counter forces. The work presented in this paper constitutes the initial study of a magnetorheological (MR) fluid-based actuator as a structural element for vibration mitigation applications. An underpinning principle for the proposed MR actuator is tuning the total structure's stiffness and damping properties by means of the MR fluid effect. The changes in stiffness and damping are observed in the form of shifts in magnitudes of the resonant natural frequency and variations in vibration amplitudes, respectively.

As well known, MR fluids change from a fluid state to a quasi-solid one when activated by a magnetic field. Such a behaviour is linked to their structure: MR fluids contain magnetic particles (usually iron) in a carrier liquid; the size of the particles ranges typically from 1 to $10 \mu \mathrm{m}$. Under the application of a magnetic field, the particles magnetise and form chains in the direction of the field lines. This rearrangement causes a non-linear increase in the apparent yield stress. With increasing field strength, MR fluids exhibit increasing resistance to flow (apparent viscosity) or increasing stiffness (elastic modulus) depending on deformation. It is generally assumed that MR fluids behave as non-Newtonian fluids in the absence of field. Under the effect of magnetic field, two types of their rheological behaviour in the pre-yield 
and post-yield regimes are modelled. In the pre-yield regime, MR fluids show linear viscoelastic behaviour that can be characterised by the complex shear modulus $\mathrm{G}^{*}$ with the storage modulus G' and loss modulus G" being its real and imaginary parts, respectively. On the other hand, the post-yield behaviour can be approximated by the Bingham plastic model. MR fluids have a short response time, of the order of a few milliseconds, and are thus suitable for real-time applications. [1]

During the last two decades, smart fluids have been investigated as structural elements for vibration mitigation. However, much research has focused on ER fluid adaptive structures compared to the work done on structures embedded with MR fluids.

The damping characteristics of ER fluid-filled cantilevered beams in free oscillation were theoretically and/or experimentally investigated by several researchers [2-6]. Other investigations on ER sandwich structures include an ER filled insert in a solid beam [7], an ER beam embedded with a fibre optic sensor [8], and cantilever ER beams rotating in the horizontal plane [9]. In all these cases, the ER structures were fully activated by homogeneous fields. Furthermore, research was done on clamped-clamped ER beams with cavities which were tested with different volumes of filling of ER fluid [10] and by activating the ER fluid in different regions of the beam [11]. In general, controllability of the ER beam was shown by changing its stiffness and damping characteristics. However, the degree of tunability was limited by the properties of the used ER fluid and hardware constraints to achieve high voltages.

Unlike the extensive literature that can be found for ER fluids structures, research on MR fluids structures is more limited. This is surprising since MR fluids have higher stiffness compared to ER fluids and therefore can facilitate higher controllability. While ER fluids achieve a yield stress between 2-5 $\mathrm{kPa}$, MR fluids achieve 50-100 $\mathrm{kPa}$ [12]. Besides, MR fluids do not need high voltage sources and have a wider range of operating temperatures. Still, an experimental work with MR fluids can be quite challenging. In ER fluid sandwich beams, the external layers of the beam (face plates) are the electrodes generating the electric field; whereas in MR fluid beams, the magnetic poles are not a part of the beam, and the magnetic field is generated externally. In a steady-state position, the magnetic field lines are perpendicular to the MR beam. However, once the MR beam is excited, it vibrates within the static magnetic field, continuously changing the angle between the magnetic field lines and the beam's axis. Moreover, since the magnetic poles are outside the beam, it might be rather demanding to generate a strong and homogeneous magnetic field at the fluid gap. In addition, the material of the outer faces should be non-magnetic so that the MR effect can be studied. Moreover, it is important to remember that the MR fluid contains magnetic particles and therefore is attracted to the magnetic poles. This could cause bending of the beam depending on the thickness and stiffness of the face plates and magnetic field strength.

Analytical models and simulations of composite structures containing MR fluids enclosed between layers can be found in literature [13-14]. The vibration characteristics of ER and MR fluid simply supported sandwich beams were experimentally investigated [15-16]. Results showed that MR materials have higher stiffness values and are recommended for vibration suppression of structures, which operate with high frequencies. However, their work presented some difficulties since their MR beam bended with the presence of magnetic field and they failed to achieve the expected magnetic field intensities. Similarly, sandwich beams with MR elastomer cores have been studied to create devices with controllable stiffness [17].

While simply-supported MR sandwich beams have been experimentally investigated before, there still lacks a body of experimental research on cantilever MR beams. So, the objective of the present work is to analyze experimentally the controllability of cantilever MR sandwich beams under different magnetic field configurations, face plates materials and amplitudes of excitation. The maximum relative change in natural frequency, and therefore in stiffness, 
obtained by adjusting the applied magnetic field is here referred as controllability. The achieved magnetic fields are stronger than those reported in literature [16]. Furthermore, this study also aims to investigate the partial activation of cantilever MR beams in specific regions.

\section{Sandwich beam design and manufacture}

A simple sandwich cantilever beam system is selected to study the performance of MR fluids in adaptive structures. Cantilever beams are frequently used to study different behaviours because of their relatively simple mechanical model and as a basis for more complex structures. In this case, the controllable capabilities of MR fluids in adaptive structures were analysed in real time. The studied cantilever beam is formed by three layers: two elastic face plates and an MR fluid core. An external magnetic field controls the rheological properties of the fluid, and hence the dynamic characteristics of the structure. Modal analysis was conducted to obtain the natural frequencies of vibration of the cantilever beam in the absence and presence of magnetic field.

It was decided to use different materials for the face plates: aluminium and polyethylene terephthalate (PET). The middle frame, made of PET in both cases, keeps a uniform MR fluid layer inside the beam. The specimens are referred to as 'aluminium beam' and 'PET beam' in this paper. Table 1 shows the general properties of the employed materials.

Table 1. Material properties

\begin{tabular}{ccc}
\hline & Density $(\rho)\left[\mathrm{kg} / \mathrm{m}^{3}\right]$ & Young's Modulus (E) $[\mathrm{GPa}]$ \\
\hline Aluminium & 2710 & 70 \\
PET & 1370 & 3 \\
MR fluid & 3000 & - \\
\hline
\end{tabular}

The MR fluid selected for this study was MRF-132DG manufactured by LORD Corporation. This hydrocarbon-based fluid has a viscosity of $0.092 \mathrm{~Pa} \cdot \mathrm{s}$ at $40^{\circ} \mathrm{C}$ and $80.98 \%$ of solids content. It contains carbonyl iron particles, which are widely used for MR fluids due to their high magnetic permeability and low coercivity, making the fluid suitable for reversible systems. Since in this specific application of the sandwich beam, the MR fluid is always in the pre-yield region, it is considered as a linear viscoelastic material.

It was decided to employ aluminium in one of the beams due to its light weight, low damping and relative high stiffness (compared to PET). Since its relative magnetic permeability is equal to unity, aluminium does not affect the strength and distribution of the magnetic field. On the other hand, the transparency of the PET face plates and frame, allowed ensuring that no air bubbles were left within the fluid during the fabrication process. Besides, the MR beam with all three layers made of PET has some benefits, such as similarity in mechanical properties of the plastic parts, glue and sealant.

Each of the two MR sandwich beams is composed of three $1 \mathrm{~mm}$ thick layers. The aluminium plates were machined and the PET parts were laser-cut to the dimensions shown in figure 1. The manufactured layers were glued together with Super Glue and sealed to avoid any leakage. Next, to be able to fill the cavity of the sandwich beam, a hole of $0.6 \mathrm{~mm}$ diameter was drilled in each side of the beam. One hole was drilled in the free end of the beam and the other one in the opposite side, very close to the clamping part. Then, the MR fluid was injected in the beam using a hypodermic syringe. Figure 2 shows a schematic of the glued MR beam and the location of the drilled holes. The hole close to the clamping part was used to let the MR fluid in and the hole in the free end was used to let the air go out. This method of filling the beam worked well without any air bubbles trapped inside. Finally, the two holes were sealed and allowed to dry. 


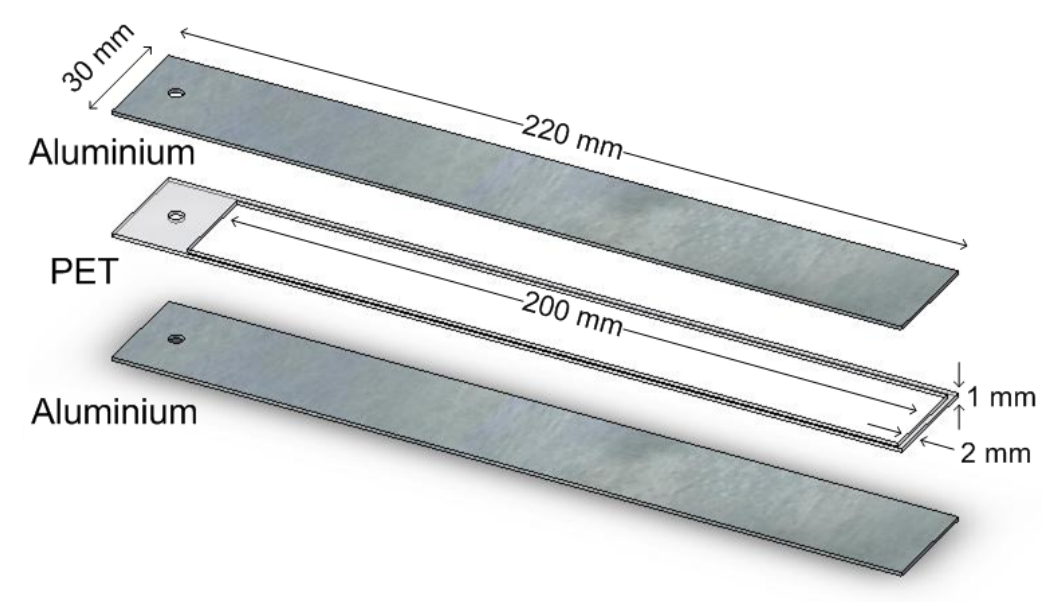

Figure 1. Design of the aluminium MR beam

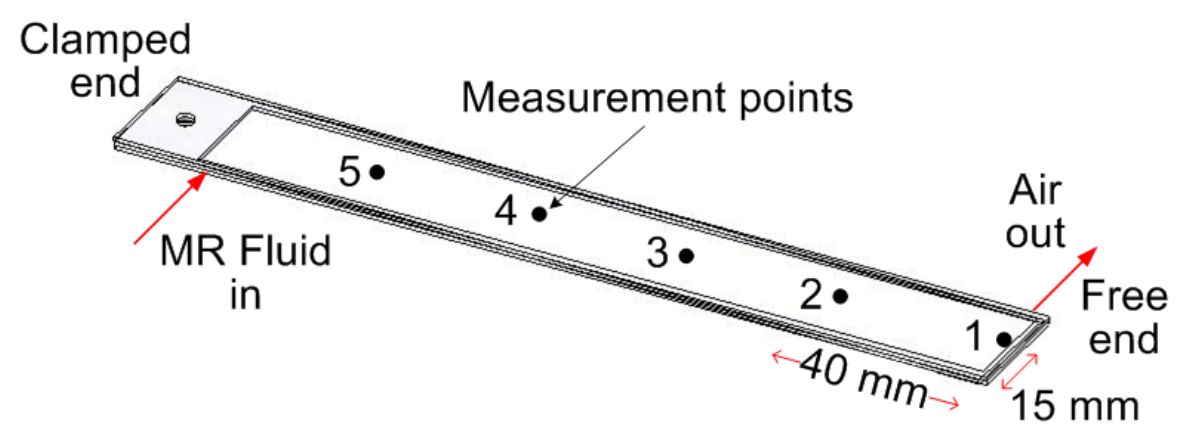

Figure 2. Glued MR beam with measurement positions 1-5 and drilled holes for injecting the MR fluid

\section{Experimental setup}

It was necessary to build a structure to clamp the MR beam for the tests. Aluminium profiles were used for a rigid base to support and hold two lines of permanent magnets and the MR beam between these magnets (figure 3). Permanent magnets were chosen for their versatility to create different magnetic field configurations (homogeneous and non-homogeneous) along the MR beam. The entire unit was mounted on a granite base for maximum measurement stability.

The main elements of the employed test rig are the structure with the cantilever MR beam and permanent magnets, an impact hammer, an amplifier, a shaker, an oscillator, a laser vibrometer, a data acquisition card and a dynamic signal analyzer. Permanent magnets generate a magnetic field, which goes through the MR beam; this field is controlled by changing the distance between the magnets. Once the MR beam is excited, the laser vibrometer measures its vibration response. The data acquisition card collects the signals from the impact hammer (if necessary) and the laser vibrometer and sends them to the dynamic signal analyzer software. Finally, the signals are processed and Fourier transformed to get the natural frequencies of the beam. A schematic of the rig is presented in figure 3 . 


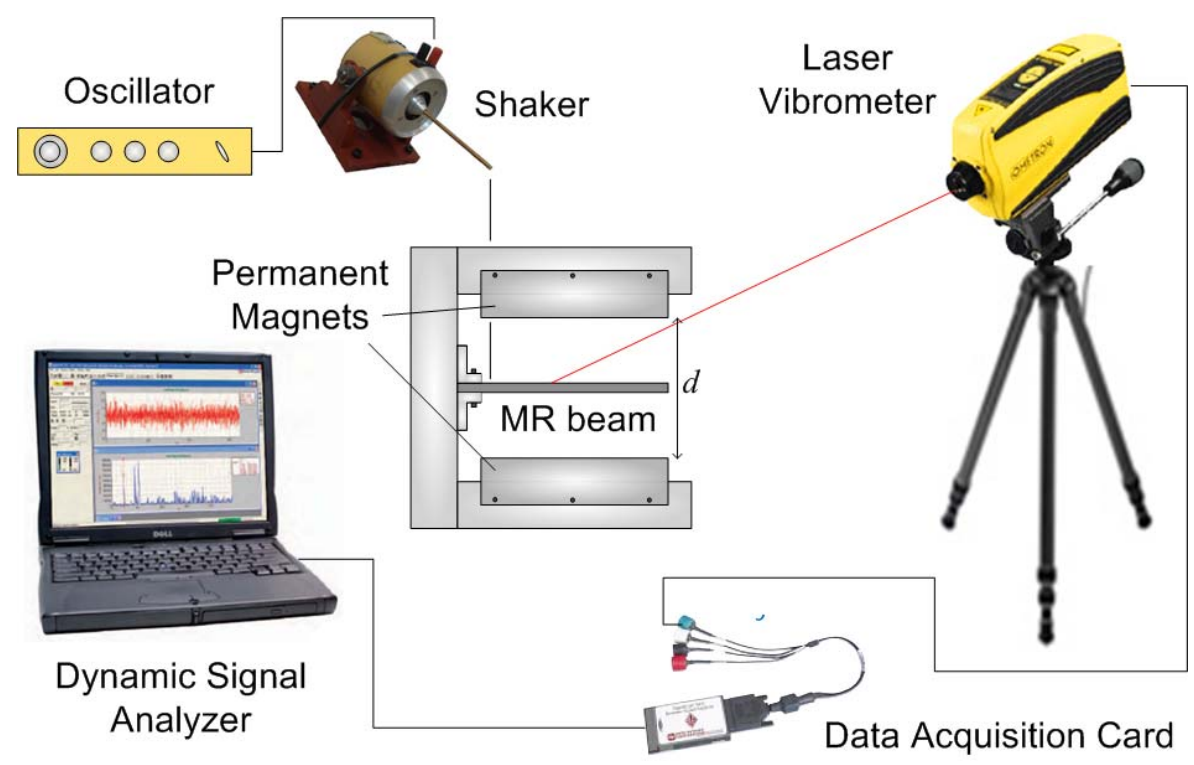

Figure 3. Schematic of the test rig

Two rows of permanent magnets were required according to the design of the test rig, since the MR beam is located between them. Different magnetic field intensities were obtained by changing the distance $d$ (figure 3) between the rows. In order to set up the magnets in the test rig, two aluminium housings were built and the Supermagnete Q404020N permanent magnets were pushed inside them (figure 4). Brackets were placed at both ends of the housing to keep the magnets in place. A hall-effect gaussmetter, Hirst model FM70, was used to identify the magnetic poles (north and south) and measure the magnetic flux generated by the magnets.

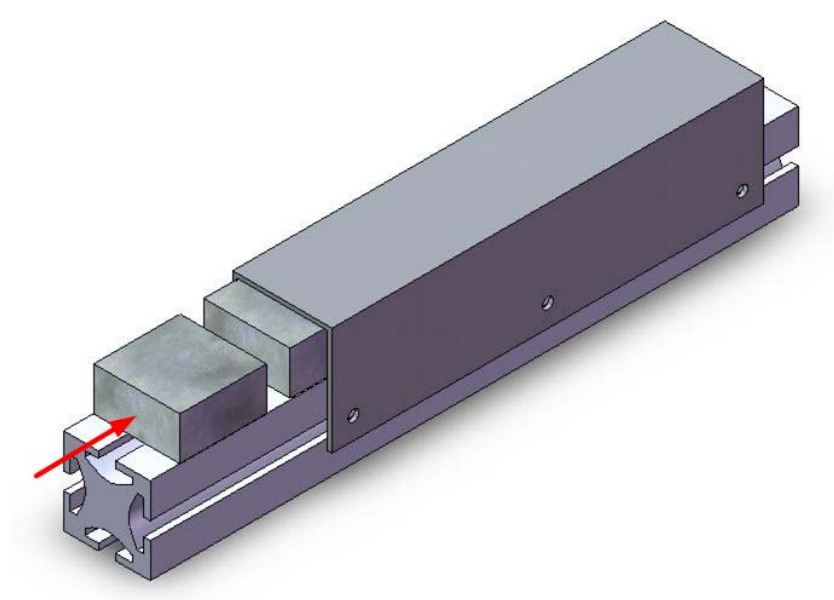

Figure 4. Setting up of permanent magnets in the test rig

Different options were employed to excite the cantilever MR beams: free vibration, impact hammer and shaker. Even though the applied forces were different in each case and the MR beam vibrated at different amplitudes because of the changes in the excitation force, the beam vibrated at the same natural frequency. For the free-vibration tests, the MR beam was released from a specific deflected set position to maintain the excitation force constant when comparing the response of the beam with and without magnetic field. 
In a modal test, usually there are two ways to produce the excitation force: an impact hammer and a shaker. Theoretically, there would not be any difference between the measured frequency response functions obtained in an impact test and those from a shaker test. However, in practice, pure forces can not be applied to a structure without any interaction between them and the structure. Moreover, the mass and stiffness effects of the shaker attachment affect the modes of the structure, and a way to compensate these effects should be found. The impact hammer used to excite the MR beam was produced by the company Brüel and Kjaer. Its tip consists of a piezoelectric force transducer, which measures the impact force, allowing compressive force measurements up to $1000 \mathrm{~N}$. The sensitivity of the impact hammer is of $22.8 \mathrm{mV} / \mathrm{N}$. A charge amplifier was used for a better signal resolution. The shaker produced by Ling Dynamic Systems is a permanent magnet electrodynamic shaker with a sine force peak of $6 \mathrm{lbf}(26.7 \mathrm{~N})$ and a displacement up to 0.2 in $(5.08 \mathrm{~mm})$. The shaker stinger was made out of brass, which is non-magnetic, and the length was enough to ensure a good performance of the shaker without any interference with the magnets of the test rig. The shaker was driven by an amplified voltage signal generated by a power oscillator by the same company, set with a swept sine actuation frequency ranging from $0 \mathrm{~Hz}$ to $200 \mathrm{~Hz}$.

The vibration response of the cantilever MR beam was measured by a laser vibrometer, the VH300 model produced by Ometron. It is a portable single-point laser Doppler vibrometer with a HeNe (Helium-Neon) laser as its source with an output of less than $1 \mathrm{~mW}$ at a wavelength of $632.8 \mathrm{~nm}$. The range of frequencies that can be detected by this sensor is from $2 \mathrm{~Hz}$ to $25 \mathrm{kHz}$ at a working distance between $0.4 \mathrm{~m}$ up to $25 \mathrm{~m}$. The maximum output signal of the vibrometer is of $\pm 10 \mathrm{~V}$ with an output sensitivity of $33.3 \mathrm{mV}$ per $\mathrm{mm} / \mathrm{s}$.

A dynamic signal analyzer was used to acquire and process the input and output signals from the system. Both the impact hammer and laser vibrometer were plugged to the SignalCalc ACE PCMCIA Data Acquisition Card, which, in turn, was connected to the computer with the SignalCalc ACE software. The dynamic analyzer includes the capability to perform the fast Fourier transformation (FFT) of the acquired analogue signals. The vibration response was obtained in the frequency domain, where natural frequencies and amplitudes of vibration were presented in the output of the analysis results.

\section{Results and discussion}

When a structure interacts with an external dynamic force, the vibration frequency response depends on the stiffness and mass distribution of the structural elements. Similarly, the magnitude of this vibration is controlled by the damping characteristics of the structure. Therefore, the vibration characteristics of the MR beams were studied by obtaining their resonant natural frequencies under different configurations of the magnetic field. Since significant shifts were presented in the resonant frequency, this study focuses on the first natural frequency of the MR beam only. Section 4.1 presents a set of preliminary tests that were conducted to define setup details for the test rig. Sections 4.2 and 4.3 describe the results obtained for the tests with the PET and aluminium MR beams, respectively.

\subsection{Preliminary tests}

In an initial set of tests, the MR beams were studied in the absence of magnetic field and the readings obtained were compared to choose the most reliable excitation way for this specific application. The readings of the first natural frequency of the PET beam corresponded to 14.844 Hz, $14.375 \mathrm{~Hz}$ and $14.531 \mathrm{~Hz}$ for the cases of free vibration, impact hammer and shaker, respectively. The data obtained for the different ways of excitation was close considering the effects of the shaker attachment on the beam. However, shaker tests were preferred to carry out the tests (with exception of free vibration tests) since the constant 
excitation force amplitude can be maintained providing more consistent results. The shaker excitation amplitude was set at a sine force peak of $13.35 \mathrm{~N}$.

The effect of the magnetic field direction on the behaviour of the MR beam was also studied. Two different magnetic field arrangements were tested with the aluminium MR beam, as schematically illustrated in figure 5. One of the arrangements suggests having adjacent magnets in alternating directions compared to the case where the magnetic field is generated in the same direction. Since equal poles repel each other and opposite poles attract, the correct orientation of the adjacent permanent magnets is confirmed by the attraction or repulsion forces, depending on the case. For a constant magnetic field magnitude, the natural frequency was tunable by $5.0 \%$ in the case of the magnetic field with alternating directions compared to the $8.1 \%$ for the same direction arrangement. It is important to consider these arrangements when working with permanent magnets, since adjacent magnetic fields with opposite directions cancel themselves in their boundaries resulting in a lower overall intensity along the beam. The latter was verified with the gaussmeter. Therefore, the arrangement of magnetic field in the same direction, figure 5(b), was chosen to carry out the tests.

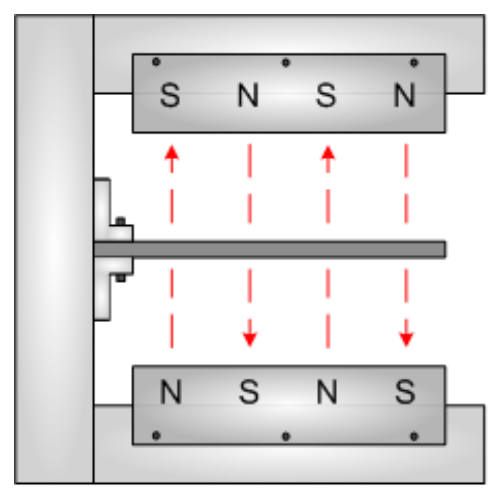

(a)

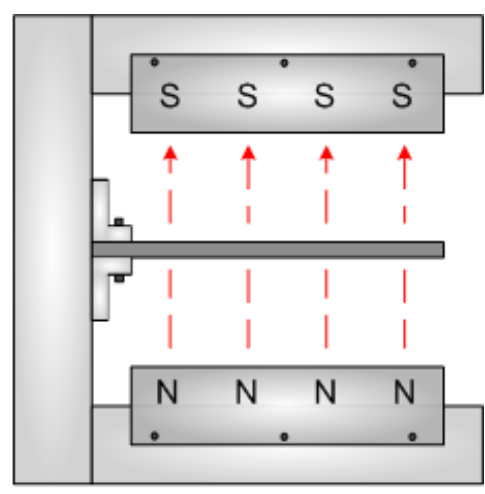

(b)

Figure 5. Magnetic field direction: (a) alternating, and (b) same direction

\subsection{PET MR beam}

The free vibration response curves of the PET MR sandwich beam are shown in figure 6 . The curves present the time response to an impulse applied at the free end of the cantilever MR beam in the absence and presence of magnetic field ( 0.11 Tesla), respectively. The data show that the beam's damping increases considerably with the applied magnetic field. From the rate of decay of the response in the free vibration plots and equations (1) and (2), the damping ratio $\xi$ can be obtained. In equation (1), $\delta$ corresponds to the $\log$ decrement, $x$ the amplitude of vibration and $n$ the number of cycles. The damping ratio in the absence of magnetic field corresponds to $0.46 \%$, and it increases to $0.65 \%$ with a field of $0.11 \mathrm{~T}$.

$$
\begin{gathered}
\delta=\frac{1}{n} \ln \frac{x_{1}}{x_{n+1}} \\
\xi=\frac{\delta}{2 \pi}
\end{gathered}
$$




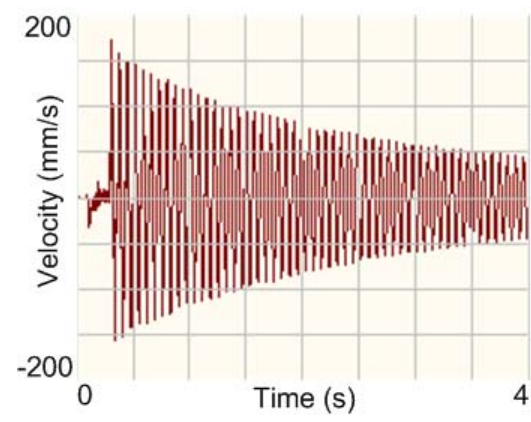

(a)

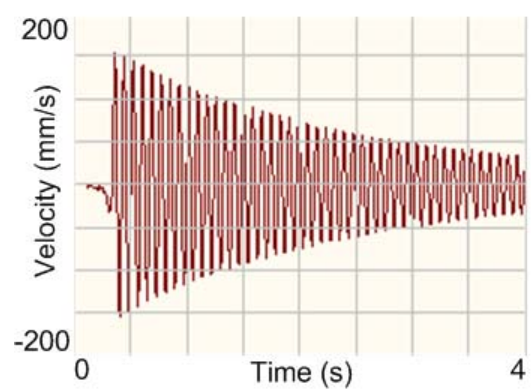

(b)

Figure 6. Free vibration response - PET MR beam: (a) without magnetic field; (b) with magnetic field

$(0.11 \mathrm{~T})$

The effect of activating the MR fluid in one specific region of the PET beam was investigated. Figure 7 shows a schematic representation of the different positions of a single Q404020N magnet along the beam. The shaker excited the beam close to the clamped end and the laser vibrometer was focused on measurement point 3 (figure 2). Figure 8 shows the obtained vibration spectra of the PET beam for different activated regions. It is obvious that the natural frequency of the beam decreases as the permanent magnet is moved away from the clamped end of the beam. This behaviour agrees with the theoretical prediction of Yalcintas and Coulter [11], where the natural frequency of a simply supported ER beam decreased when the beam was activated only in the central regions, away from the clamped ends. Stiffening the fluid in the regions away from the clamps of the beam, results in a decrease in the natural frequency of the beam compared with the natural frequency in the absence of field. Major changes in frequency are observed as the magnet moves towards the free end.

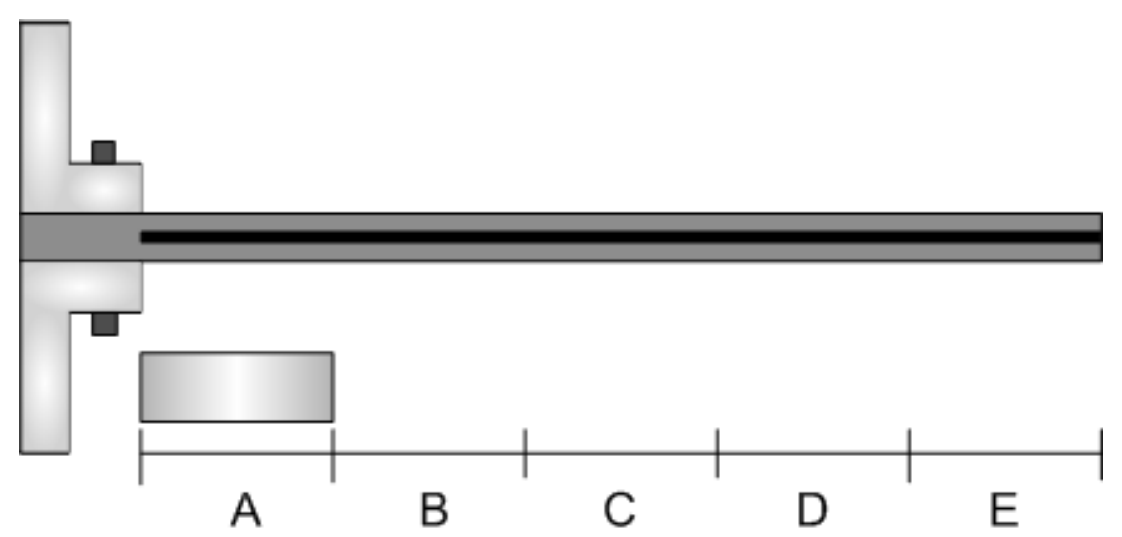

Figure 7. Magnetic field applied in specific regions of the MR beam 


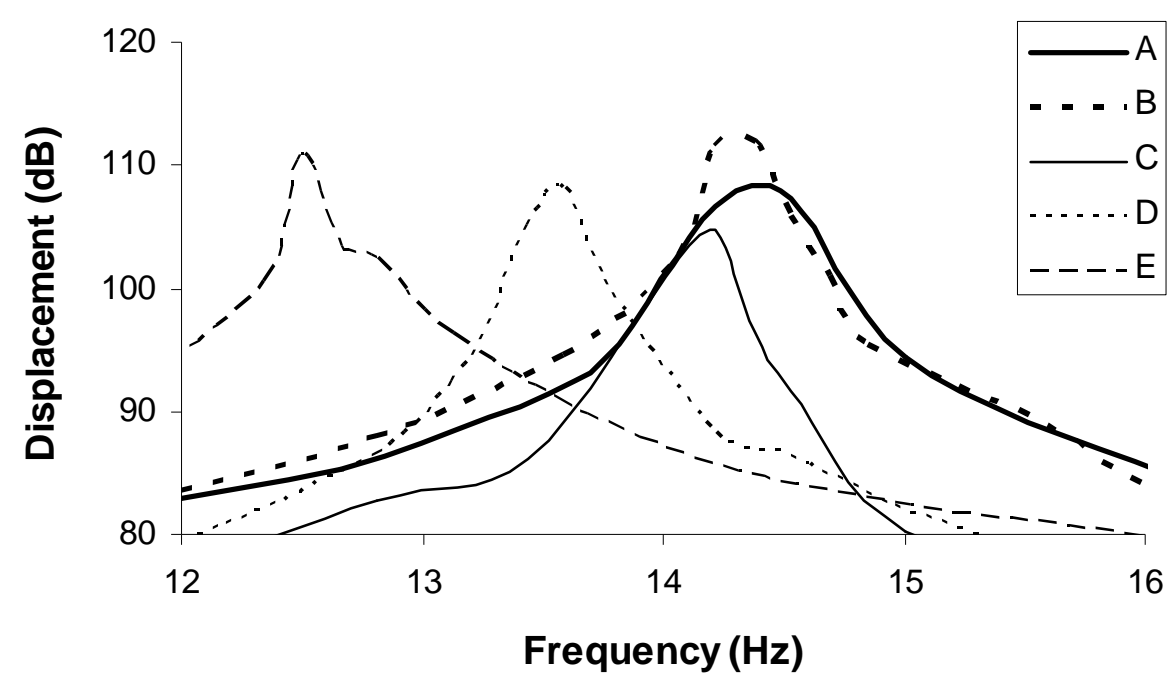

Figure 8. Vibration spectra of PET MR beam activated at different regions

The PET beam was also investigated under different non-homogeneous magnetic field levels and configurations. In all the cases, the distance between the magnetic poles corresponded to $6.9 \mathrm{~cm}$. The variations of magnetic field intensities were obtained by varying the number of permanent magnets within the aluminium housings, as shown in table 2. All the magnets inside an aluminium housing have the same polarity and therefore they repel each other; as a consequence, the gaps between the magnets are equal. These gaps cause the non-homogeneity of the magnetic field since it is not uniform in magnitude along the beam. Figure 9 illustrates the change in the resonant frequency of the PET beam upon the application of the magnetic field. It can be observed that for stronger magnetic fields, the resonant natural frequencies are shifted to lower frequencies with smaller vibration levels. This behaviour might be due to the mass concentration of the iron particles in specific places and the non-homogeneous stiffening of the MR fluid along the beam. The PET beam's natural frequencies were experimentally tuned up to $26.9 \%$ and the vibration levels decreased as much as $2.33 \mathrm{~dB}$. Measurements with stronger magnetic fields could not be achieved with the PET beam since it was not stiff enough and it was being attracted to the magnets.

Table 2. Effects of arrangement of magnets on levels of non-homogeneous magnetic fields

\begin{tabular}{cc}
\hline Amount of magnets in the housings (upper and lower) & Magnetic field strength $[\mathrm{T}]$ \\
\hline no magnets & 0 \\
3 lower only & 0.05 \\
3 upper and 3 lower & 0.08 \\
4 upper and 4 lower & 0.11 \\
5 upper and 5 lower & 0.14 \\
\hline
\end{tabular}




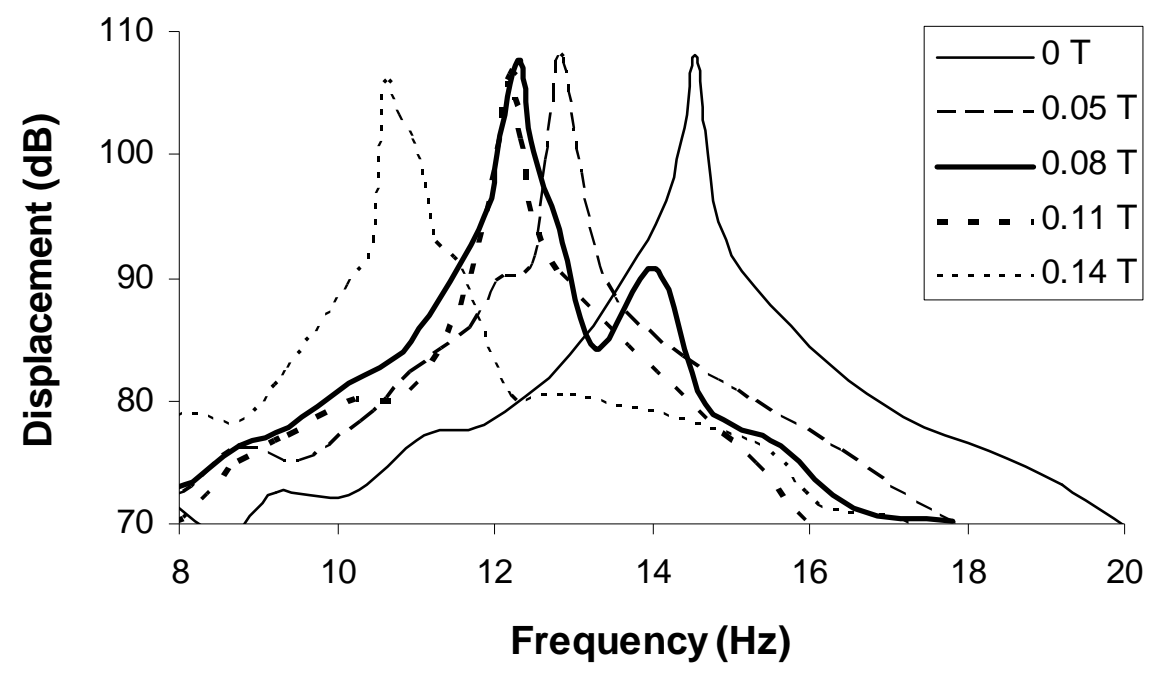

Figure 9. First natural frequency of PET beam for different levels of non-homogeneous magnetic fields

\subsection{Aluminium MR beam}

The free vibration response curves of the aluminium MR sandwich beam are shown in figure 10. The tests were conducted in a similar way that with the PET beam. However, a magnetic field of $0.23 \mathrm{~T}$ was applied since the aluminium beam is stiffer and withstands stronger magnetic fields without bending. The free vibration plots clearly show that the beam's damping increases significantly with the applied magnetic field. The calculated damping ratio in the absence of magnetic field for the aluminium beam corresponds to $1.8 \%$, and it increases to $3.7 \%$ with a field of $0.23 \mathrm{~T}$.

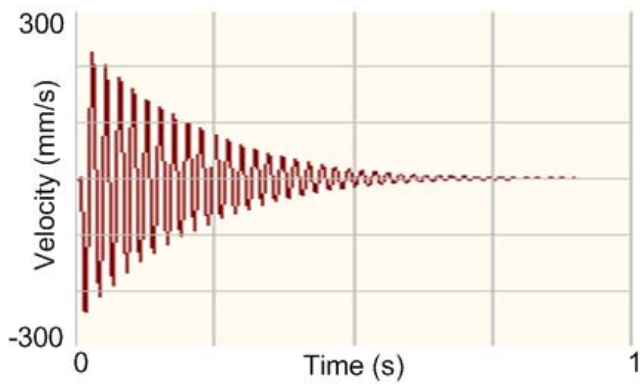

(a)

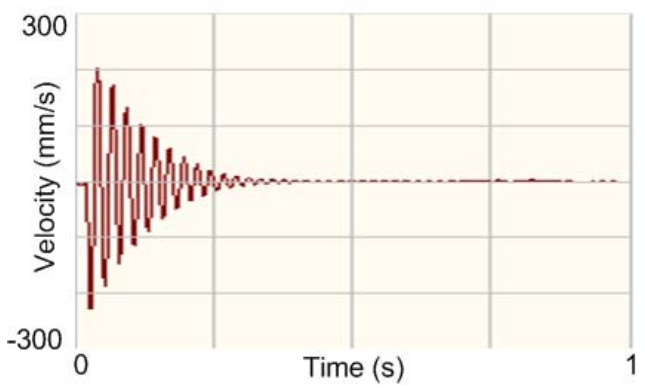

(b)

Figure 10. Free vibration response - aluminium MR beam: (a) without magnetic field; (b) with magnetic field $(0.23 \mathrm{~T})$ 
The natural frequency of the aluminium MR beam was measured for a variety of nonhomogeneous magnetic fields. The magnetic fields of $0 \mathrm{~T}, 0.11 \mathrm{~T}$ and $0.14 \mathrm{~T}$ were generated with the arrangements described in table 2. In order to generate the magnetic field of $0.24 \mathrm{~T}$, eight Supermagnete Q252513N magnets were added to the arrangement used for $0.14 \mathrm{~T}$. Four magnets were placed on top of each aluminium housing and were attached by magnetic forces to the magnets inside the housing (figure 11). Different readings were obtained when these extra magnets were placed either closer to the clamped end or to the free end. Figure 12 shows the obtained first natural frequency of the aluminium MR beam. There is a clear effect on the vibration properties of the aluminium beam. Once again, the natural frequency of the MR beam is shifted to lower magnitudes by activating the MR fluid only in some regions of the beam. For stronger magnetic fields, a bigger decrement in frequencies is observed. Moreover, when applying the magnetic field closer to the free end, a higher controllability can be observed than when applying it closer to the clamped one. Such behaviour was also observed for the PET beam. With a magnetic field of $0.24 \mathrm{~T}$ closer to the free end, the aluminium beam was tunable by $17.5 \%$ of the initial natural frequency.

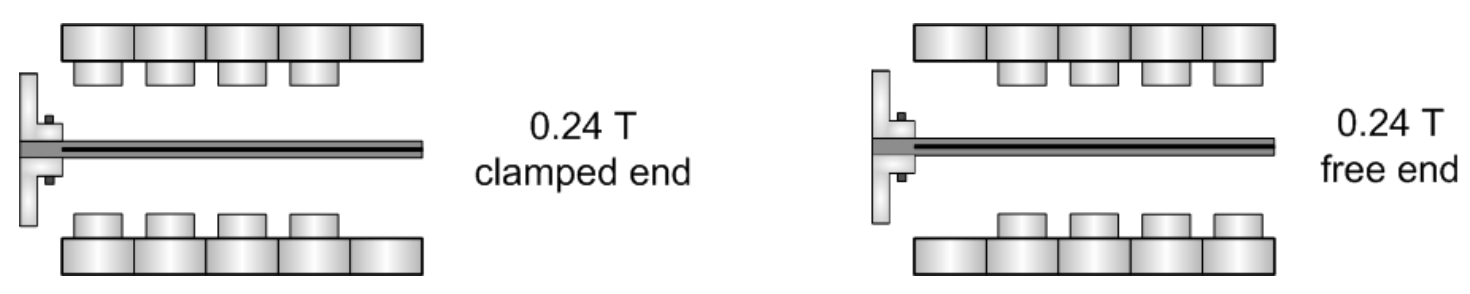

Figure 11. Attachment of Q252513N magnets

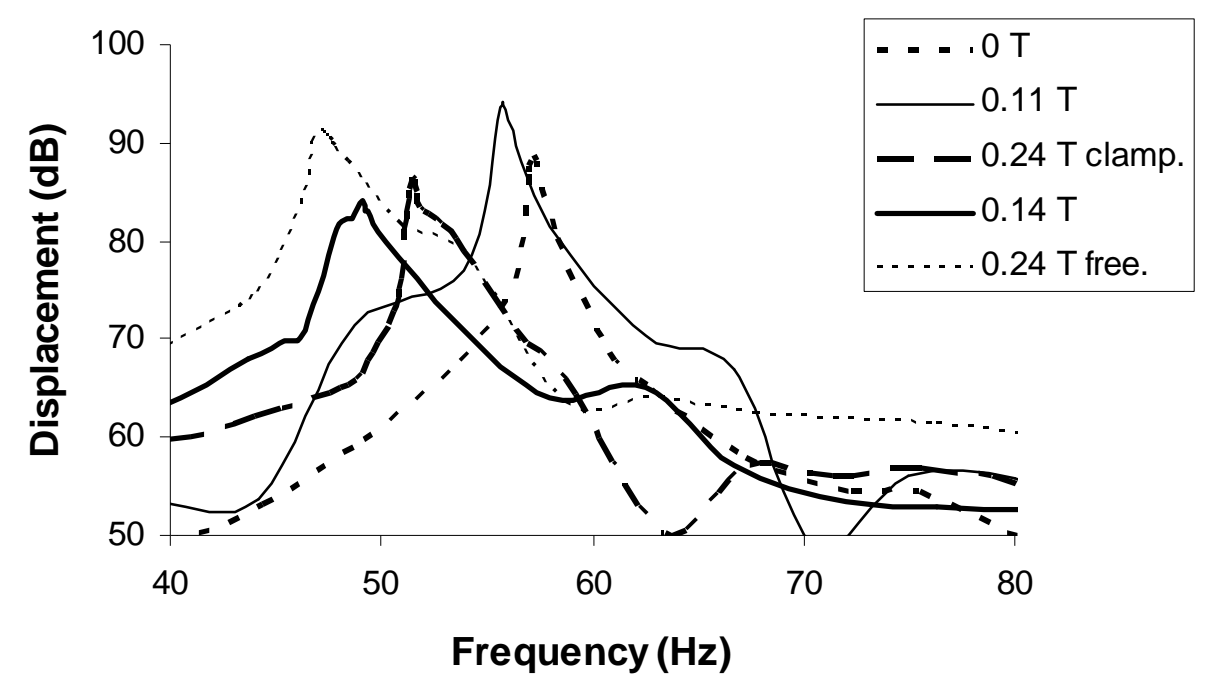

Figure 12. First natural frequency of aluminium beam at different values of non-homogeneous magnetic fields 
For another set of tests, the test rig was modified to bring the magnetic poles closer to a distance of $4.8 \mathrm{~cm}$ in order to achieve a stronger and more homogeneous magnetic field of $0.23 \mathrm{~T}$. Five permanent magnets were pushed together in each aluminium housing. The increasing stiffening effect of the aluminium MR beam was observed with this new arrangement. An increase in the first natural frequency of $10.5 \%$ was observed as compared to the case without the field. By decreasing the distance between the magnets, a more homogeneous magnetic field was generated with the same amount of magnets. It was observed that there is a certain distance between the magnetic poles where the damping effect on the beam becomes greater than the stiffening effect. It is hypothesised that in low homogeneous magnetic fields, the storage modulus G' is smaller than the loss modulus G" resulting in highly effective damping in the structure and low vibration amplitudes; whilst with stronger magnetic fields G' becomes greater than G', so the stiffening effect overcomes the damping effect resulting in higher vibration amplitudes. It can be also supposed that in stronger magnetic fields the attraction force between the MR beam and the magnetic poles plays a significant role in its vibration response. Further research is needed to explore these conjectures.

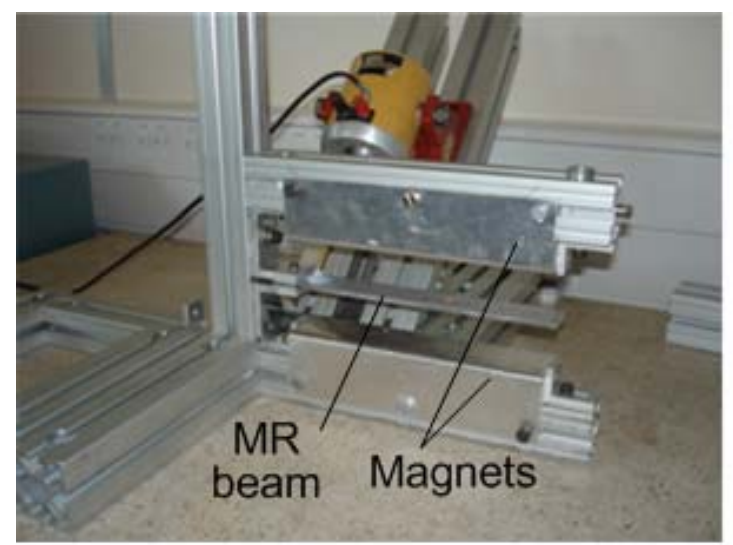

(a)

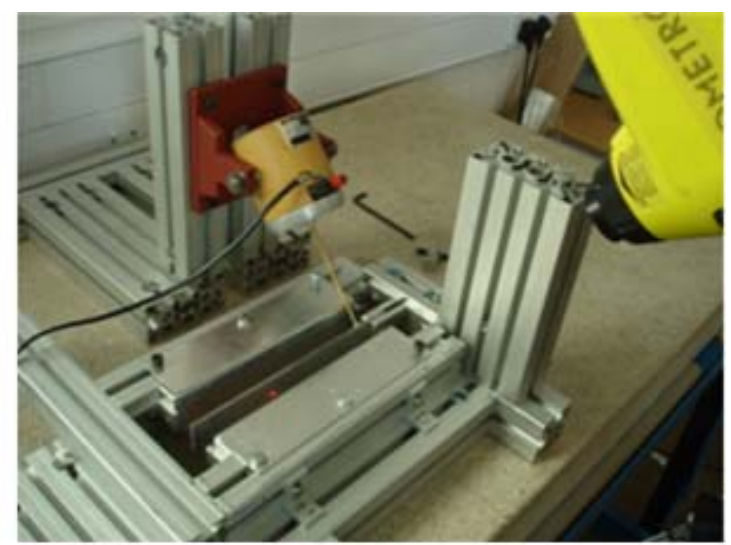

(b)

Figure 13. Test rig with horizontal (a) and vertical (b) MR beam configurations

Finally, the MR cantilever beam was clamped in two different configurations: horizontal and vertical (figure 13). The former (figure 13a) was used for most of the tests conducted. The vertical beam configuration allowed reducing the effect of gravity on the MR beam. In that configuration, the effect of two different locations of the magnetic poles was studied: parallel and perpendicular to the wide face of the MR beam. In the first case, the magnetic field was generated uniformly all along the MR beam. In the latter, the edges of the MR beam were closer to the magnets; hence, the magnetic field was stronger there than in the central part of the beam.

The stiffening effect of the magnetic field in the aluminium MR beam is demonstrated in figure 14. The aluminium beam was clamped in the vertical configuration. The frequency response shows the change in the natural frequency of the beam when the homogeneous magnetic field is applied along the beam. When the magnetic poles were parallel to the wide face of the aluminium MR beam, a magnetic field of $0.23 \mathrm{~T}$ was generated resulting in the increase in the natural frequency by $5.9 \%$. At the same time, there was an improvement in the damping properties of the MR beam since the vibration level decreased significantly; the vibration amplitude decreased $15.7 \mathrm{~dB}$. The test rig was then modified to place the magnetic poles perpendicularly to the wide face of the aluminium MR beam. Because the distance between the magnetic poles was the same, a stronger magnetic field was achieved on the sides of the aluminium beam $(0.32 \mathrm{~T})$, resulting in the increase of $15.9 \%$ for the first natural frequency. 


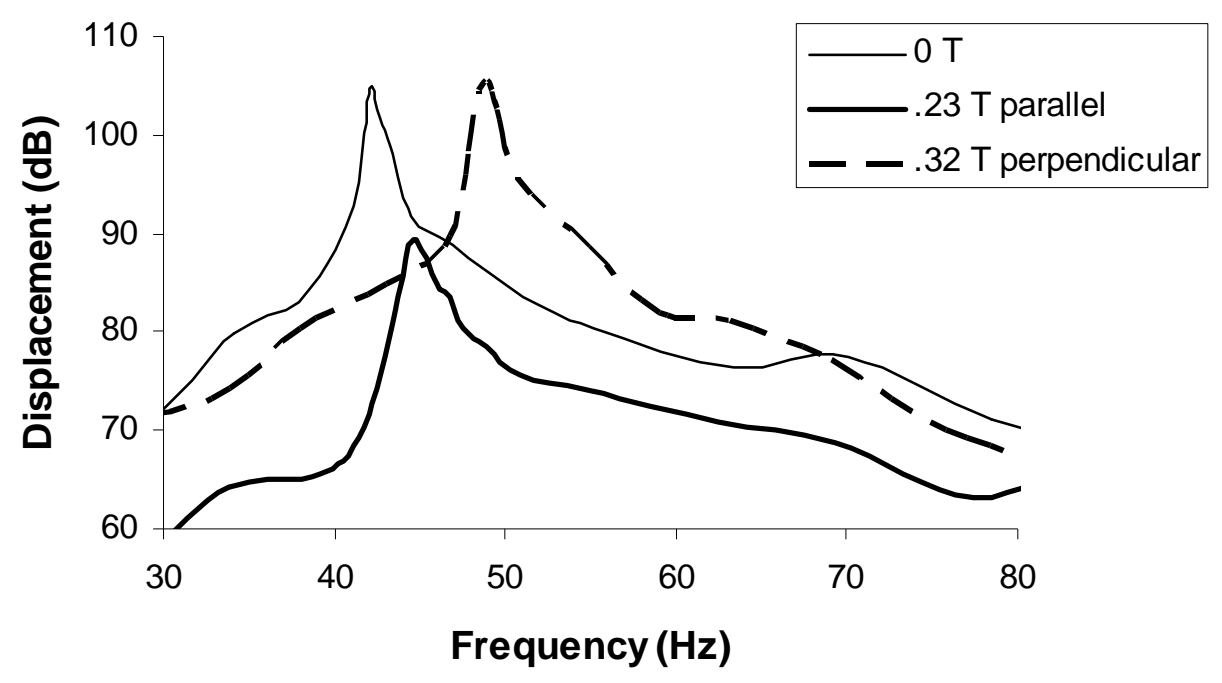

Figure 14. Stiffening effect in aluminium MR beam

The changes in natural frequencies can be compared for the horizontal and vertical configurations for the same magnetic field intensity and distribution, i.e. $0.23 \mathrm{~T}$ with magnetic poles parallel to the wide face of the MR beam. The natural frequency increased by $10.93 \%$ in the first case compared to the $5.93 \%$ obtained for the vertical configuration. Several factors could have an effect on these results, such as gravity, attraction forces between the beam and the magnets, the angle of the shaker stinger, shaker attachment, and weight distribution of the aluminium-profiles structure. The next stage of investigations will deal with analysis of these factors.

\section{Conclusions}

In this paper an experimental approach to study the tunability of MR fluid-filled beams was described. The particular case of cantilever sandwiched beam was considered, but the principle can be applied to more complex structures. The stiffness and damping characteristics of the MR beams were investigated as a function of different magnetic field configurations and intensities. The beams were tested in free vibration and with an excitation force generated either with an electrodynamic shaker or an impact hammer. Shaker tests were preferred since the excitation force amplitude can be maintained constant.

\section{The observed vibration characteristics of the MR beams are summarized as follows:}

(1) MR beams were fabricated with two different materials for comparison purposes. The PET beam showed bigger shifts in natural frequencies than the aluminium beam for a same magnetic field level and configuration. This was expected since the stiffness of aluminium is much higher than that of PET. This should be taken into account while deciding which material to use for a specific application.

(2) The effect of factors such as magnetic field direction, magnetic field homogeneity, mass distribution and excitation force amplitude resulted imperative in order to obtain the expected response. While typical research done on ER and MR fluid structures suggests shifting the resonant natural frequency of the structure to higher frequencies, the present paper also suggests the possibility of shifting this frequency to lower values. It was demonstrated that this can be achieved by the partial activation of the beam, i.e. applying the magnetic field only to some sections of the beam. Unlike ER fluids, MR fluids are activated magnetically so there is no need for dividing the structure in cavities to be able to activate selected regions of the beam only. In some cases, the partial activation of the MR beam results in smaller vibration amplitudes than when the beam is fully activated. 
This opens the possibility of developing more energy-efficient designs resulting in an adequate controllability of the natural vibration frequencies and amplitudes according to the specific application.

(3) The experimental study revealed the tunability of the stiffness and damping characteristics of MR beams. The PET beam exhibited a change in frequency up to $26.9 \%$ and variations in vibration amplitude of as much as $2.3 \mathrm{~dB}$. Likewise, the natural frequency of the aluminium beam was tuned to achieve variations of $17.5 \%$ and the vibration amplitude was decreased by as much as $15.7 \mathrm{~dB}$.

Future work would be done to study the loss and storage moduli effects on the vibration response of the structure under a range of homogeneous magnetic field intensities.

\section{Acknowledgements}

The authors wish to acknowledge the supports from EPSRC and CONACYT. The valuable assistance of the Wolfson school technicians and MRG students during the set-up of the test rig is also highly appreciated.

\section{References}

[1] Carlson J D, Matthis W and Toscano J R 2001 Smart prosthetics based on magnetorheological fluids SPIE 8th Annual Symp. on Smart Struct. Mater. 2001 (Newport Beach CA, USA)

[2] Ghandi M V, Thompson B S and Choi S B 1989 A new generation of innovative ultraadvanced intelligent composite materials featuring electro-rheological fluids: an experimental investigation J. Composite Mater. 23 1232-1255

[3] Choi Y, Sprecher A F and Conrad H 1990 Vibration characteristics of a composite beam containing an electrorheological fluid J. Intell. Mater. Syst. Struct. 1 91-104

[4] Berg C D 1996 Composite structure analysis of a hollow cantilever beam filled with electro-rheological fluid J. Intell. Mater. Syst. Struct. 7 494-502

[5] Lee C Y 1995 Finite element formulation of a sandwich beam with embedded electrorheological fluids J. Intell. Mater. Syst. Struct. 6 718-728

[6] Phani A S and Venkatraman K 2003 Vibration control of sandwich beams using electrorheological fluids Mech. Syst. and Signal Processing 17 1083-95

[7] Harland N R, Mace B R and Jones R W 2001 Adaptive-passive control of vibration transmission in beams using electro/magnetorheological fluid filled inserts IEEE Transactions on Control Systems Technology 9 209-20

[8] Leng J and Asundi A 1999 Active vibration control system of smart structures based on FOS and ER actuator J. Smart Mater. Struct. 8 252-6

[9] Wei K, Meng G, Zhou S and Liu J 2006 Vibration control of variable/speed acceleration rotating beams using smart materials. J. of Sound and Vibration 298 1150-8

[10] Oyadiji S O 1996 Applications of electro-rheological fluids for constrained layer damping treatment of structures J. Intell. Mater. Syst. Struct. 7 541-9

[11] Yalcintas M and Coulter J P 1998 Electrorheological material based non-homogeneous adaptive beams J. Smart Mater. Struct. 7 128-43

[12] Carlson D, Catanzarite D M and Clair K A 1996 Commercial magneto-rheological fluid devices Proc. 5th Int. Conf. on ER Fluids, MR Suspensions and Associated Technology 1995 (Sheffield, UK: World Scientific) pp 20-8

[13] Weiss K D, Duclos T G, Chrzan M J and Yanyo L C 1996 Magnetorheological fluid composite structures US Patent 5,547,049

[14] Chen L and Tian J 2006 Distributed magnetorheological (MR) fluid damper for active structural vibration control Proc. of Active 2006: Int. Symp. on Active Control of Sound and Vibration ed C Q Howard and M R F Kidner (Adelaide, AU) 
[15] Yalcintas M and Dai H 1999 Magnetorheological and electrorheological materials in adaptive structures and their performance comparison J. Smart Mater. Struct. 8 560-73

[16] Yalcintas M and Dai H 2004 Vibration suppression capabilities of magnetorheological materials based adaptive structures J. Smart Mater. Struct. 13 1-11

[17] Zhou G Y and Wang Q 2005 Magnetorheological elastomer-based smart sandwich beams with nonconductive skins J. Smart Mater. Struct. 14 1001-9 